\title{
Stem Cell Therapy for Neurodegenerative Diseases: How Do Stem Cells Bypass the Blood-Brain Barrier and Home to the Brain?
}

\author{
Yvonne Cashinn Chia, ${ }^{1,2}$ Clarice Evey Anjum,, ${ }^{1,2}$ Hui Rong Yee, ${ }^{1,2}$ Yenny Kenisi, ${ }^{1,2}$ \\ Mike K. S. Chan, ${ }^{1,2}$ Michelle B. F. Wong, ${ }^{1,2}$ and Shing Yi Pan (id) \\ ${ }^{1}$ Baden ReD Laboratories GmbH, Germany \\ ${ }^{2}$ Baden Research and Testing (Asia Pac) Sdn Bhd, Malaysia \\ Correspondence should be addressed to Shing Yi Pan; dr.pan@badenresearch.com
}

Received 21 May 2020; Revised 11 August 2020; Accepted 20 August 2020; Published 4 September 2020

Academic Editor: Renke Li

Copyright (c) 2020 Yvonne Cashinn Chia et al. This is an open access article distributed under the Creative Commons Attribution License, which permits unrestricted use, distribution, and reproduction in any medium, provided the original work is properly cited.

\begin{abstract}
Blood-brain barrier (BBB) is a term describing the highly selective barrier formed by the endothelial cells (ECs) of the central nervous system (CNS) homeostasis by restricting movement across the BBB. An intact BBB is critical for normal brain functions as it maintains brain homeostasis, modulates immune cell transport, and provides protection against pathogens and other foreign substances. However, it also prevents drugs from entering the CNS to treat neurodegenerative diseases. Stem cells, on the other hand, have been reported to bypass the BBB and successfully home to their target in the brain and initiate repair, making them a promising approach in cellular therapy, especially those related to neurodegenerative disease. This review article discusses the mechanism behind the successful homing of stem cells to the brain, their potential role as a drug delivery vehicle, and their applications in neurodegenerative diseases.
\end{abstract}

\section{Introduction}

The blood-brain barrier (BBB) is a selective barrier formed by the central nervous system (CNS) endothelial cells (ECs) which are connected by continuous tight junctions, creating a restricted movement of molecules across the BBB. The physiological barrier is regulated by a series of physical, transport, and metabolic properties as well as interactions with different vascular, immune, and neural cells [1]. Apart from junction proteins, the BBB is comprised of adherence proteins, transporters, basal lamina, and extracellular matrix at the molecular level [2]. The BBB is surrounded by endothelial cells, pericytes, astrocytic foot processes, neurons, mast cells, microglia, and circulating immune cells [3-5].

The selective barrier capacity of $\mathrm{BBB}$ allows it to regulate CNS homeostasis and protect the CNS against toxins, pathogens, inflammation, injury, and disease $[1,6]$. However, disruption or dysregulation of the BBB may cause changes in permeability that permit pathogens into the brain or cause neuroinflammation, consequently contributing towards the progression of several CNS-related diseases including neurodegenerative diseases (e.g., Parkinson's disease and Alzheimer's disease), cerebrovascular disease (e.g., stroke), and traumatic brain injuries [7-11].

In general, there are two pathways that allow molecular movement across the BBB: first, through free diffusion via lipid solubility and, second, through catalysed transport [12]. Through extensive studies, researchers have found that compounds that are fat-soluble and/or very small in sizes, such as alcohol and certain low molecular weight narcotics or hormones (i.e., below 400-500 Dalton), can easily pass through the ECs that make up the BBB via the transcellular pathway $[13,14]$. In contrast, specific transporters or receptors are necessary to facilitate the movement of various other larger nutrients, ions, and macromolecules [15]. Due to the restrictive characteristic of the $\mathrm{BBB}$, there is an obstacle for drug delivery to the brain. An estimated $98 \%$ of potential therapeutics for brain disorders fail to penetrate the $\mathrm{BBB}$ 
$[16,17]$. In light of these challenges, efforts have been made to create strategies to modulate and bypass the BBB so that life-saving drugs can reach specific targets in the brain without disturbing other ongoing activities [18].

It is vital to understand the mechanism of $\mathrm{BBB}$ regulation in treating neurological diseases as many CNS therapeutics are unable to bypass the $\mathrm{BBB}$. The use of stem cells to bypass the BBB for CNS-related disease treatment has been promising. While stem cells are most commonly delivered via direct transplantation which allows nonsystemic homing, they often cause injury at the site of injection. In contrast, the administration of stem cell through intravenous, intramuscular, or intranasal does not cause injury, but stem cells must systemically home and bypass the BBB. This review article further explains the homing mechanism of stem cells bypassing the $\mathrm{BBB}$ and the applications of stem cell therapy in neurodegenerative diseases.

\section{How Do Stem Cells Pass Through the BBB and Home?}

Many different stem cells can be used in the treatment of neurological diseases, including but not limited to mesenchymal, neural, and embryonic stem cells. For the sake of discussion in this review, we will be citing MSCs as an example, considering it is the most widely used stem cells in brainrelated treatment. The ability to protect damaged tissue and differentiate into a range of cells by secreting trophic, immune-modulatory, or other engineered therapeutic factors makes them an excellent choice in cellular therapy [19-21]. Nevertheless, the BBB remains a major physical barrier that MSCs and other stem cells must overcome to reach their targeted site in the brain and exert their therapeutic effects [4]. Unfortunately, no detailed studies have been conducted yet on how much the BBB is an active barrier for MSCs in treating the brain [22].

However, studies have shown that MSCs are capable of migrating across endothelial cells by either the paracellular or transcellular pathway and subsequently preferentially home back to the site of inflammation or injury in the brain to exert their therapeutic effects $[23,24]$. This has been demonstrated in a whole-body imaging study in mouse using MSC-labelled fluorescence magnetic nanoparticles [25]. While the mechanism of how endogenous MSCs migrate and function in response to injury remains poorly understood, certain injuries such as traumatic brain injury, stroke, brain tumour, or aging are believed to compromise the efficiency of BBB protection $[8,9,26,27]$. This allows MSCs to migrate across the endothelial cells via paracellular pathways through the formation of a transient interendothelial gap [23]. It has been postulated that activation of endothelial cells and astrocytes in some of these CNS injuries causes lower tight junction integrity and formation of paracellular gaps which allow cell migration via the paracellular route [20]. In addition, MSCs are also capable of abolishing and splitting tight junctions between endothelial cells [24].

Homing of MSCs could happen either systemically or nonsystemically. In nonsystemic homing, MSCs are transplanted directly at the target tissue and subsequently guided by chemokines and other factors to the site of injury. In contrast, in systemic homing, MSCs are administered away from the site target tissue or site of injury. Scientist proposed that the systemic homing of MSCs results from their interaction with endothelial cells in a leukocyte-like, multistep cascade which eventually allows them to migrate across the BBB. In general, several different mechanism models proposed that MSCs travel through the circulatory system and subsequently leave the blood circulation by integrating into the endothelium, transmigrating through the endothelial barrier, and penetrating the basement membrane before invading the tissue via the formation of plasmic podia [28]. The multistep cascade of MSC homing mechanism as described by Ullah et al. ([29]) consists of five different steps, namely, (1) tethering and rolling, (2) activation by cytokines, (3) cell arrest by integrins, (4) transmigration, and (5) migration, and is illustrated in Figure 1. Liu et al. [20] discussed a similar but simplified homing mechanism, which includes rolling, adhesion, and transmigration.

MSCs first enter the bloodstream when they are therapeutically administered. The homing process begins with MSCs decelerating and coming into contact with the endothelial wall by tethering to the selectins expressed by endothelial cells and starts to roll along the vasculature wall [30, 31]. Next, integrin receptors, like VLA-4, is activated in response to G-protein-coupled chemokine receptors such as stromal cell-derived factor-1 (SDF-) 1 , which binds ligands expressed by MSCs such as CXCR4 or CXCR7 [29, 30]. Following activation of integrin, MSCs arrest on the endothelial membrane as integrins like VLA- 4 expressed by MSC bind with VCAM-1 on endothelial cells [32]. The earlier activation increases the affinity of integrins essential for cell arrest; hence, the VLA-4/VCAM-1 interaction allows MSCs to adhere to the endothelial cells firmly [29, 32]. Next, MSCs travel through the endothelial cell layer and basement membrane in a process called transmigration, facilitated by the secretion of matrix metalloproteinases (MMPs) which degrades the endothelial basement membrane [28, 33, 34]. Alternatively, abolishment and splitting of the tight junctions between endothelial cells by MSC could also facilitate their transmigrations [24]. MSCs have also been reported to penetrate the endothelia via plasmic podia $[28,34]$. Finally, MSCs migrate to the site of injury, guided by various signals released by the damaged tissue, such as growth factors and chemokines [29, 35]. Once at the target side, MSCs can induce modification of the damaged tissue microenvironment to promote regeneration and protection [36].

The delivery method is thought to influence the homing ability of stem cells. Direct transplantation at the site of injury, for example, through intracerebral injection or using microcannula, is thought to be a more efficient approach for a successful homing of the cell, although they may cause injury at the site of injection [37]. On the other hand, indirect delivery, especially through intravenous injection, has been reported to cause MSCs to be entrapped in the lung vasculature [38]. Nevertheless, over time, these MSCs eventually migrate from the lungs to other tissues, including the brain $[25,32]$. However, entrapment in the lung may shorten the therapeutic life and potential effect of MSCs [39]. Literature 


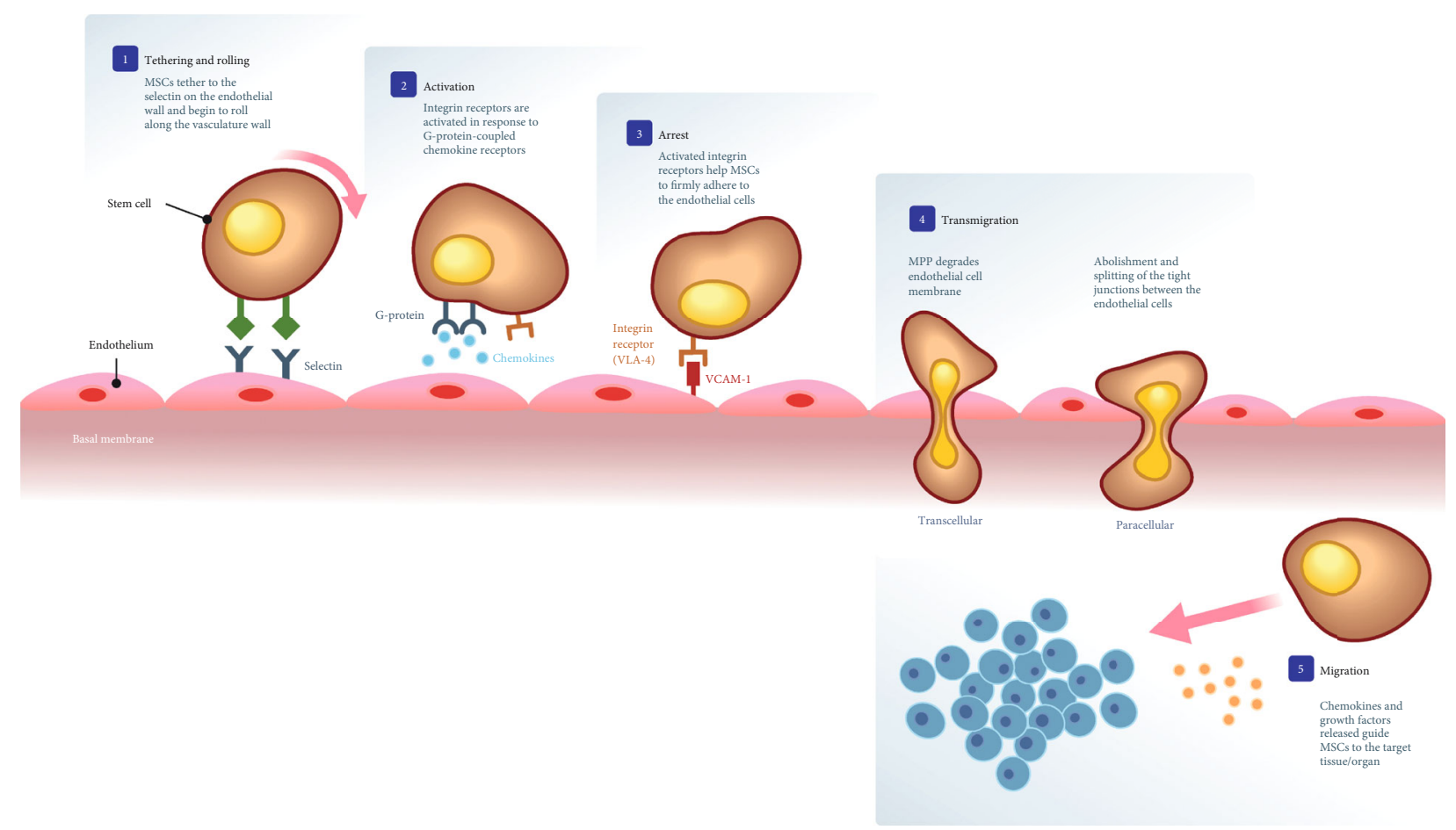

FIGURE 1: The multistep cascade of MSC homing mechanism consists of five different steps, namely, (1) tethering and rolling, (2) activation by cytokines, (3) cell arrest by integrins, (4) transmigration, and (5) migration.

suggests that intramuscular injection is becoming a popular method of MSC delivery, with promising results seen in the treatment of several paediatric neurological disabilities including muscular dystrophy and cerebral palsy [40]. In contrast to the short therapeutic life of MSCs delivered via intravenous injection, intramuscular injection of MSCs could potentially treat both distant or systemic conditions with sustained benefit owing to the longer dwelling time of active secretory cells and has been clinically proven to be safe [39, 41]. Alternatively, stem cells may be delivered via intranasal application, a cheap and easy alternative route found to be effective in bypassing the BBB for the treatment of neurodegenerative disorders [42, 43]. However, to this day, there is still no consensus on how stem cells used in the treatment of neurodegenerative disease should be delivered.

\section{Stem Cell Therapy as a Treatment Modality for Neurodegenerative Diseases}

The integrity of the BBB, influenced by disease severity and duration, is believed to contribute towards the progression of certain neurodegenerative diseases, although their mechanism remains unclear [44]. However, studies have shown that stem cell therapy represents a promising treatment modality in tissue regeneration and repair for many central nervous system or neurodegenerative diseases [45]. The use of stem cells like MSCs or neural stem cells has been showing promising results, with potential to slow down and, in some cases, reverse the progress of some of these neurodegenerative diseases [46-49]. Treatment with MSCs, in particular, has been very popular, likely due to their neuroprotective and immunomodulatory properties in which neurotrophic and growth factors are released by MSCs to promote tissue repair and regeneration [50]. As discussed in the previous section, stem cells are capable of homing back to the side of injury to initiate endogenous repair. Furthermore, MSCs are thought to mediate multiple mechanisms of actions, making them useful in the treatment of a wide range of diseases [51]. Interestingly, transplantation of MSCs showed the absence of cell replacement evidence, suggesting transient recovery may be induced by trophic effects $[22,36]$. The application of MSCs has been extensively studied both in many animal models and some clinical studies, including but not limited to Parkinson's disease (PD), Alzheimer's disease (AD), amyotrophic lateral sclerosis (ALS), multiple sclerosis, and stroke [48]. Nevertheless, stem cells as a therapeutic tool against neurodegenerative disease comes with many benefits and challenges as summarised in Table 1.

$\mathrm{PD}$ is a neurodegenerative disease characterised by loss of dopaminergic cells in the substantia nigra, although it may also be caused by degeneration of other neurotransmission systems [21]. Hence, treatments that prevent the loss of dying neuronal cells or transplantation of cells with neuronal properties are imperative in managing PD [52]. Studies of the PD in both human and animal models have shown that MSCs could reverse parkinsonian symptoms [46, 47]. While the exact mechanism behind the improvement is unclear, the authors postulated that it is likely that better dopaminergic regulation could have resulted from the survival and functioning of transformed dopaminergic neurons and their terminals [47]. Follow-up of up to 36 months in the same small clinical study, in which autologous bone marrowderived MSCs were transplanted by stereotaxic surgery, suggests that treatment with MSCs is relatively safe, without 
TABLE 1: Benefit and limitation of stem cells as a therapeutical tool in neurodegenerative diseases.

\begin{tabular}{|c|c|}
\hline Benefit & Limitation \\
\hline $\begin{array}{l}\text { (i) Possess neuroprotective and immunomodulatory } \\
\text { properties which promote tissue repair and regeneration }\end{array}$ & $\begin{array}{l}\text { (i) Underlying mechanism of action remains unclear and will require more } \\
\text { detailed investigations }\end{array}$ \\
\hline (ii) Capable of migrating across the endothelial cell of $\mathrm{BBB}$ & $\begin{array}{l}\text { (ii) Must successfully overcome the BBB in order to exert therapeutic effects } \\
\text { in the brain }\end{array}$ \\
\hline $\begin{array}{l}\text { (iii) Capable of homing back to the side of injury to initiates } \\
\text { endogenous repair }\end{array}$ & (iii) Homing potential is influenced by the delivery method \\
\hline $\begin{array}{l}\text { (iv) Absence of cell replacement evidence; trophic effects } \\
\text { likely induce transient recovery }\end{array}$ & $\begin{array}{l}\text { (iv) Sufficient numbers of cells reaching the target site are necessary to exert a } \\
\text { therapeutical effect }\end{array}$ \\
\hline $\begin{array}{l}\text { (v) Generally well-accepted with no serious adverse effects } \\
\text { such as infection or tumour }\end{array}$ & $\begin{array}{l}\text { (v) Treatment outcome may be affected by various factors including the } \\
\text { donor's age, host tissue, and growth regulators expressed by recipient tissue }\end{array}$ \\
\hline
\end{tabular}

signs of tumour formation or other adverse side effects [47]. Another study suggests MSCs may have a neuroprotective effect on dopaminergic neurons via anti-inflammatory action, which promotes recovery of BBB integrity [53]. In addition, research also suggests MSCs could stabilise the permeability of the BBB by modulating astrocytic endfeet and vascular endothelial growth factor A (VEGF-A) signalling in patients with PD [44].

$\mathrm{AD}$ is the most common form of dementia, with currently no definite cure [54]. This disorder is pathologically characterised by the deposition of amyloid- $\beta$ peptide and the formation of neurofibrillary tangles in affected brain regions [55]. The efficacy of MSCs in treating AD has been demonstrated in preclinical models in several studies. The feasibility of using bone marrow-derived MSCs as a therapeutic agent in an acutely induced AD mouse model has been tested by injecting MSC into the dentate gyrus of the hippocampus of the mouse [56]. The study concluded that the bone marrow-derived MSCs could increase microglia activation and promote the reduction of amyloid- $\beta$ peptide in the brain of the $\mathrm{AD}$ model. In an $\mathrm{AD}$-related environment, autophagy is essential as it plays a critical role in maintaining neuronal homeostasis [57]. MSCs were found to enhance autophagy and exerted a neuroprotective effect by modulating amyloid- $\beta$ clearance in the AD mouse model. This finding suggests that a damaged $\mathrm{AD}$ brain could potentially be repaired by using MSCs through the modulation of the autophagy pathway [58]. The safety and tolerability of MSCs as a treatment for AD have been affirmed in phase 1 clinical trial. In the clinical trial, umbilical cord blood-derived MSCs were delivered via intraparenchymal administration in nine patients with mild-to-moderate $\mathrm{AD}$. However, the efficacy of the treatment was not established due to the small sample size [59].

ALS or known as Lou Gehrig's disease is a neurological disorder with no known cure, but stem cell therapies, particularly MSCs, are a promising candidate for ALS treatment. Preclinical work in mouse models of ALS suggests that bone marrow-derived MSCs can be induced to secrete neurotrophic factors (NTFs) which delay motor neuron degeneration and improve motor performance $[60,61]$. Based on this data, intramuscular (IM) implantation of bone marrow MSC-NTFs in ALS patients has been pursued in a phase $1 / 2$ (NCT01051882) and 2a (NCT01777646) clinical trials
[62]. In the phase $1 / 2$ of the trial, six ALS patients in early stages were administered with MSC-NTFs via the IM method and another six patients with advanced stages of ALS were injected intrathecally (IT). The phase 2 a clinical trial, which is a dose-escalating study, involved a total of 14 early-stage ALS patients who received a combination of IM and IT transplantation of autologous MSC-NTFs. IM implantation of MSC-NTFs in an ALS rat model study was previously shown to ameliorate motor neuron loss which occurs in the initial stage of ALS [63]. Meanwhile, MSCs which are injected through IT implantation have a greater chance of migrating to the proximity of the CNS lesions [64]. In the clinical trial, no serious adverse effects were reported following the IM, IT, and IM+IT transplantation of MSC-NTFs although some patients experienced headache, fever, vomiting, leg and back pain, and neck stiffness. Other than that, no infection nor tumour formation was found on the site of injection. Although IM implantation of MSC-NTFs only induced a minor beneficial clinical effect compared to IT implantation, both methods are safe with indications of possible, clinically meaningful benefits in patients with ALS [62].

Traumatic brain injuries (TBI) have been found to trigger neurodegeneration, which elevates the risk of developing into a more serious condition such as chronic traumatic encephalopathy, dementia, $\mathrm{AD}$, and $\mathrm{PD}$ if left untreated $[8,65]$. Increasing evidence supporting the efficiency of using MSCs in alleviating TBI sequelae has begun to emerge in recent years, suggesting that MSCs could enhance the function of a patients' nervous system. Anbari et al. have demonstrated that the intravenously administered MSCs in rats with TBI were able to differentiate into neuron- and astrocyte-like cells which then improve sensory and motor function and enhance neural growth and regeneration [66]. Another study conducted by Cox et al. has intravenously implanted MSCs into 10 children that had TBI with a Glasgow Coma Scale (CGS) score between 5 and 8 and monitored them for 6 months [67]. Among the 10 children, 7 of them showed positive results with improvements on the GCS. Another 3 children did not show significant improvement in their quality of life. However, none of them suffered from any adverse effects or died due to the use of MSC therapy. Subsequently, MSCs were used to treat TBI in adults with positive results [68], proving MSC-based approaches could serve as treatments for patients who are suffering from TBI. 


\section{Mesenchymal Stem Cells as Delivery Vehicles for Antitumour Agents}

Besides their endogenous therapeutic properties, there have been increasing interest in the potential of MSCs to be used as a delivery vehicle. In this review, we will be focusing on the potential of MSCs as a drug delivery vehicle for antitumour agents, especially by leveraging on the tumourhoming ability of MSCs. Besides its inherent tumour-tropic property, MSCs are also a desirable delivery vehicle due to their immune-modulatory capabilities [30]. Several types of antiglioma agents have been studied as MSC cargoes such as prodrug enzyme, secreted proteins, oncolytic viruses, and nanoparticles.

Administration of MSCs expressing prodrug-converting gene is a promising experimental approach in glioblastoma treatment. For this purpose, MSCs are first genetically modified to express a prodrug-converting enzyme. Upon injecting back into the body, the MSCs will migrate towards the tumour cells. Subsequently, when a prodrug is administered, it will be converted into its active cytotoxic form by the enzyme expressed by the genetically modified MSCs. This in turn produces a bystander effect, causing the death of the stem cells and concurrently killing the surrounding tumour cells [69-71]. In this strategy, the homing ability of MSCs allows it to deliver a local high-dose active chemotherapeutic agent to the tumour without causing systemic toxicity which is observed in many chemotherapeutic agents [72]. Currently, thymidine kinase/ganciclovir (TK/GCV) and yeast cytosine deaminase/5-fluorocytosine (yCD/5-FC) are the most widely used enzyme/prodrug system in the literature. For instance, herpes simplex virus thymidine kinase (HSVtk) prodrug-activating gene therapy approaches have been employed in phase III clinical trial to study its therapeutic effects in glioblastoma multiforme (GBM) patients. However, the study reported no significant differences in terms of survival rate between the treated group and the control group, which may be caused by a poor rate of delivery of the HSV-tk gene to tumour cells [73].

MSCs can also be engineered to secrete protein either through transduction with a replication-incompetent viral vector carrying gene encoding protein or transfection with a gene-carrying plasmid [31]. The gene will be transcribed and translated into protein and subsequently secreted from the MSC to affect the tumour. In this case, the engineered MSC acts as a "pump" of the secreted protein within the tumour [74]. Cytokines such as interleukin are an example of secreted proteins that have been successfully delivered by MSCs in animal glioma model systems. For instance, a preclinical study in a mouse model showed that MSCs integrated with interleukin-2 were able to migrate towards the periphery of the glioma after two weeks of MSC intracerebral injection [75]. Besides interleukin, another study showed that human MSCs secreting IFN-beta were capable of homing to gliomas following intravascular injection [76].

Additionally, MSCs can be used to deliver oncolytic viruses to gliomas. As the MSCs home to the tumour, they are capable of concealing the viruses from the immune system, although the exact mechanism is not fully understood
[77]. Once loaded inside the MSCs, the virus undergoes replication and will infect and destroy the tumour cells upon release [77, 78]. Furthermore, MSCs loaded with a novel oncolytic virus named Delta-24-RGD (also known as DNX2401) have been shown to selectively localise to the glioma cell, resulting in improved tumour eradication in subsets of mice [79]. The virus is currently being studied in numerous phase 1 and 2 clinical trials for patients with recurrent GBM; hence, results regarding its safety and efficacy are largely unpublished yet [80].

Researchers have also attempted to home MSC-bearing nanoparticles to tumour tissue in the brain. Nanoparticles have several limitations when they are administered systemically as they are diluted upon penetrating the BBB, resulting in their low concentration in the brain and have unfavourable pharmacokinetic properties [81]. Hence, the integration of nanoparticles into the MSCs is believed to circumvent this problem. A study by Roger et al. [82] has shown that polylactic acid nanoparticles and lipid nanocapsules efficiently fused into the MSCs without affecting the viability and differentiation of the cells and subsequently migrate towards human glioma xenografts. In another study, Wang et al. [83] reported that MSCs loaded with paclitaxel-poly(lactic-coglycolic acid) nanoparticles were able to induce tumour cell death in orthotopic glioma rats with a little effect on the MSC migration capacity, cell cycle, or multilineagedifferentiation potential.

Despite the promising potential of MSC-mediated delivery of antiglioma agents, there are still challenges that need to be overcome. Firstly, the modification of MSCs bearing antitumour agents should not alter its tumour-homing capacity [30]. It is also important to put into consideration that different sources of MSCs may have different abilities in promoting or suppressing the growth of glioma cells under different conditions. In addition, the cargoes must not prematurely kill the MSC and should only be released from the MSC once within the tumour [72]. Further studies are highly required as there is still much discrepancy in terms of the efficiency of the combination of these antiglioma agents and MSCs. Lastly, a better understanding of the biological consequences of using MSC as delivery agents for antiglioma agents is of utmost importance before it can be widely applied for treating patients with malignant gliomas.

\section{Conclusion}

Treatment with stem cells thus far presents promising potential in treating patients with neurodegenerative diseases, with many clinical safety and efficacy experiences. The affinity and ability of stem cells to bypass the BBB and migrate to the brain makes them a versatile treatment modality, either by relying on the endogenous therapeutic properties of stem cells or by leveraging on their homing property for drug delivery purposes. Nevertheless, more research is necessary to understand the underlying mechanism of action better and to broaden the application of stem cells as a therapeutical tool in the treatment of neurodegenerative diseases. 


\section{Conflicts of Interest}

The authors declare no conflict of interest.

\section{Acknowledgments}

The authors would like to thank Foong C. C. for the illustration used in this publication.

\section{References}

[1] R. Daneman and A. Prat, "The blood-brain barrier," Cold Spring Harbor Perspectives in Biology, vol. 7, no. 1, article a020412, 2015.

[2] K. E. Warren, "Beyond the blood:brain barrier: the importance of central nervous system (CNS) pharmacokinetics for the treatment of CNS tumors, including diffuse intrinsic pontine glioma," Frontiers in Oncology, vol. 8, p. 239, 2018.

[3] N. J. Abbott, "Astrocyte-endothelial interactions and bloodbrain barrier permeability," Journal of Anatomy, vol. 200, no. 6, pp. 629-638, 2002.

[4] P. Ballabh, A. Braun, and M. Nedergaard, "The blood-brain barrier: an overview," Neurobiology of Disease, vol. 16, no. 1, pp. 1-13, 2004.

[5] W. A. Banks, "From blood-brain barrier to blood-brain interface: new opportunities for CNS drug delivery," Nature Reviews Drug Discovery, vol. 15, no. 4, pp. 275-292, 2016.

[6] N. J. Abbott, A. A. K. Patabendige, D. E. M. Dolman, S. R. Yusof, and D. J. Begley, "Structure and function of the blood-brain barrier," Neurobiology of Disease, vol. 37, no. 1, pp. 13-25, 2010.

[7] B. S. Desai, A. J. Monahan, P. M. Carvey, and B. Hendey, "Blood-brain barrier pathology in Alzheimer's and Parkinson's disease: implications for drug therapy," Cell Transplantation, vol. 16, no. 3, pp. 285-299, 2007.

[8] N. S. N. Graham and D. J. Sharp, "Understanding neurodegeneration after traumatic brain injury: from mechanisms to clinical trials in dementia," Journal of Neurology, Neurosurgery \& Psychiatry, vol. 90, no. 11, pp. 1221-1233, 2019.

[9] B. W. McColl, N. J. Rothwell, and S. M. Allan, "Systemic inflammation alters the kinetics of cerebrovascular tight junction disruption after experimental stroke in mice," Journal of Neuroscience, vol. 28, no. 38, pp. 9451-9462, 2008.

[10] N. Weiss, F. Miller, S. Cazaubon, and P.-O. Couraud, "The blood-brain barrier in brain homeostasis and neurological diseases," Biochimica et Biophysica Acta (BBA) - Biomembranes, vol. 1788, no. 4, pp. 842-857, 2009.

[11] A. Wong, Y. Mao, A. Levy, J. Rothstein, D. Bergles, and P. C. Searson, "The blood-brain barrier: an engineering perspective," Frontiers in Neuroengineering, vol. 6, p. 7, 2013.

[12] W. M. Pardridge, "Transport of small molecules through the blood-brain barrier: biology and methodology," Advanced Drug Delivery Reviews, vol. 15, no. 1-3, pp. 5-36, 1995.

[13] L. Cucullo, B. Aumayr, E. Rapp, and D. Janigro, "Drug delivery and in vitro models of the blood-brain barrier," Current Opinion in Drug Discovery \& Development, vol. 8, no. 1, pp. 89-99, 2005.

[14] W. M. Pardridge, "Drug and gene targeting to the brain with molecular Trojan horses," Nature Reviews Drug Discovery, vol. 1, no. 2, pp. 131-139, 2002.
[15] S. Gupta, S. Dhanda, and R. Sandhir, "Anatomy and physiology of blood-brain barrier," in Brain Targeted Drug Delivery System, pp. 7-31, Elsevier, 2019.

[16] A. Dove, "Breaching the barrier," Nature Biotechnology, vol. 26, no. 11, pp. 1213-1215, 2008.

[17] W. M. Pardridge, "The blood-brain barrier: bottleneck in brain drug development," NeuroRX, vol. 2, no. 1, pp. 3-14, 2005.

[18] J. Larsen, D. Martin, and M. Byrne, "Recent advances in delivery through the blood-brain barrier," Current Topics in Medicinal Chemistry, vol. 14, no. 9, pp. 1148-1160, 2014.

[19] D. Lo Furno, G. Mannino, and R. Giuffrida, "Functional role of mesenchymal stem cells in the treatment of chronic neurodegenerative diseases," Journal of Cellular Physiology, vol. 233, no. 5, pp. 3982-3999, 2018.

[20] L. Liu, M. A. Eckert, H. Riazifar, D.-K. Kang, D. Agalliu, and W. Zhao, "From blood to the brain: can systemically transplanted mesenchymal stem cells cross the blood-brain barrier?," Stem Cells International, vol. 2013, Article ID 435093, 7 pages, 2013.

[21] N. A. Salem, "Mesenchymal stem cell based therapy for Parkinson's disease," International Journal of Stem Cell Research \& Therapy, vol. 6, no. 1, p. 62, 2019.

[22] P. Conaty, L. S. Sherman, Y. Naaldijk, H. Ulrich, A. Stolzing, and P. Rameshwar, "Methods of mesenchymal stem cell homing to the blood-brain barrier," in Somatic Stem Cells, pp. 81-91, Humana Press, 2018.

[23] T. Matsushita, T. Kibayashi, T. Katayama et al., "Mesenchymal stem cells transmigrate across brain microvascular endothelial cell monolayers through transiently formed inter-endothelial gaps," Neuroscience Letters, vol. 502, no. 1, pp. 41-45, 2011.

[24] A. Schmidt, D. Ladage, C. Steingen et al., "Mesenchymal stem cells transmigrate over the endothelial barrier," European Journal of Cell Biology, vol. 85, no. 11, pp. 1179-1188, 2006.

[25] S. Kim, K.-A. Chang, J. a. Kim et al., "The preventive and therapeutic effects of intravenous human adipose-derived stem cells in Alzheimer's disease mice," PLoS One, vol. 7, no. 9, article e45757, 2012.

[26] C. D. Arvanitis, G. B. Ferraro, and R. K. Jain, "The blood-brain barrier and blood-tumour barrier in brain tumours and metastases," Nature Reviews Cancer, vol. 20, no. 1, pp. 26-41, 2020.

[27] M. Erickson and W. Banks, "Age-associated changes in the immune system and blood-brain barrier functions," International Journal of Molecular Sciences, vol. 20, no. 7, p. 1632, 2019.

[28] C. Steingen, F. Brenig, L. Baumgartner, J. Schmidt, A. Schmidt, and W. Bloch, "Characterization of key mechanisms in transmigration and invasion of mesenchymal stem cells," Journal of Molecular and Cellular Cardiology, vol. 44, no. 6, pp. 10721084, 2008.

[29] M. Ullah, D. D. Liu, and A. S. Thakor, "Mesenchymal stromal cell homing: mechanisms and strategies for improvement," iScience, vol. 15, pp. 421-438, 2019.

[30] L. Labusca, D. D. Herea, and K. Mashayekhi, "Stem cells as delivery vehicles for regenerative medicine-challenges and perspectives," World Journal of Stem Cells, vol. 10, no. 5, pp. 43-56, 2018.

[31] R. Sackstein, J. S. Merzaban, D. W. Cain et al., "Ex vivo glycan engineering of CD44 programs human multipotent mesenchymal stromal cell trafficking to bone," Nature Medicine, vol. 14, no. 2, pp. 181-187, 2008. 
[32] B. Rüster, S. Göttig, R. J. Ludwig et al., "Mesenchymal stem cells display coordinated rolling and adhesion behavior on endothelial cells," Blood, vol. 108, no. 12, pp. 3938-3944, 2006.

[33] A. BeckerDe, P. Van Hummelen, M. Bakkus et al., "Migration of culture-expanded human mesenchymal stem cells through bone marrow endothelium is regulated by matrix metalloproteinase- 2 and tissue inhibitor of metalloproteinase-3," Haematologica, vol. 92, no. 4, pp. 440-449, 2007.

[34] G. Chamberlain, H. Smith, G. E. Rainger, and J. Middleton, "Mesenchymal stem cells exhibit firm adhesion, crawling, spreading and transmigration across aortic endothelial cells: effects of chemokines and shear," PLoS One, vol. 6, no. 9, article e25663, 2011.

[35] L. Zachar, D. Bačenková, and J. Rosocha, "Activation, homing, and role of the mesenchymal stem cells in the inflammatory environment," Journal of Inflammation Research, vol. Volume 9, pp. 231-240, 2016.

[36] N. Joyce, G. Annett, L. Wirthlin, S. Olson, G. Bauer, and J. A. Nolta, "Mesenchymal stem cells for the treatment of neurodegenerative disease," Regenerative Medicine, vol. 5, no. 6, pp. 933-946, 2010.

[37] M. G. Cunningham, H. Bolay, C. W. Scouten et al., "Preclinical evaluation of a novel intracerebral microinjection instrument permitting electrophysiologically guided delivery of therapeutics," Neurosurgery, vol. 54, no. 6, pp. 1497-1507, 2004.

[38] U. M. Fischer, M. T. Harting, F. Jimenez et al., "Pulmonary passage is a major obstacle for intravenous stem cell delivery: the pulmonary first-pass effect," Stem Cells and Development, vol. 18, no. 5, pp. 683-692, 2009.

[39] L. R. Braid, C. A. Wood, D. M. Wiese, and B. N. Ford, "Intramuscular administration potentiates extended dwell time of mesenchymal stromal cells compared to other routes," Cytotherapy, vol. 20, no. 2, pp. 232-244, 2018.

[40] A. Sharma, H. Sane, N. Gokulchandran et al., "Stem cell therapy in pediatric neurological disabilities," Physical Disabilities - Therapeutic Implications, vol. 117, 2017.

[41] S. H. Jahromi and J. E. Davies, "Concise review: skeletal muscle as a delivery route for mesenchymal stromal cells," Stem Cells Translational Medicine, vol. 8, no. 5, pp. 456-465, 2019.

[42] L. R. Hanson and W. H. Frey II, "Intranasal delivery bypasses the blood-brain barrier to target therapeutic agents to the central nervous system and treat neurodegenerative disease," BMC Neuroscience, vol. 9, no. S3, article S5, 2008.

[43] M. Salama, M. Sobh, M. Emam et al., "Effect of intranasal stem cell administration on the nigrostriatal system in a mouse model of Parkinson's disease," Experimental and Therapeutic Medicine, vol. 13, no. 3, pp. 976-982, 2017.

[44] H. J. Park, J. Y. Shin, H. N. Kim, O. Se Hee, S. K. Song, and P. H. Lee, "Mesenchymal stem cells stabilize the blood-brain barrier through regulation of astrocytes," Stem Cell Research \& Therapy, vol. 6, no. 1, p. 187, 2015.

[45] F. Vincent, "Stem cells in tissue repair and regeneration," The Journal of Investigative Dermatology, vol. 132, no. 6, pp. 15381541, 2012.

[46] A. Kumar, S. Dudhal, A. S. T et al., "Dopaminergic-primed fetal liver mesenchymal stromal-like cells can reverse parkinsonian symptoms in 6-hydroxydopamine-lesioned mice," Cytotherapy, vol. 18, no. 3, pp. 307-319, 2016.

[47] N. K. Venkataramana, S. K. V. Kumar, S. Balaraju et al., "Open-labeled study of unilateral autologous bone-marrowderived mesenchymal stem cell transplantation in Parkinson's disease," Translational Research, vol. 155, no. 2, pp. 62-70, 2010.

[48] R. Volkman and D. Offen, "Concise review: mesenchymal stem cells in neurodegenerative diseases," Stem Cells, vol. 35, no. 8, pp. 1867-1880, 2017.

[49] T. Yasuhara, N. Matsukawa, K. Hara et al., "Transplantation of human neural stem cells exerts neuroprotection in a rat model of Parkinson's disease," Journal of Neuroscience, vol. 26, no. 48, pp. 12497-12511, 2006.

[50] T. Tanna and V. Sachan, "Mesenchymal stem cells: potential in treatment of neurodegenerative diseases," Current Stem Cell Research \& Therapy, vol. 9, no. 6, pp. 513-521, 2014.

[51] M. T. Turnbull, A. C. Zubair, J. F. Meschia, and W. D. Freeman, "Mesenchymal stem cells for hemorrhagic stroke: status of preclinical and clinical research," NPJ Regenerative Medicine, vol. 4, no. 1, 2019.

[52] M. Kitada and M. Dezawa, "Parkinson's disease and mesenchymal stem cells: potential for cell-based therapy," Parkinson's Disease, vol. 2012, Article ID 873706, 9 pages, 2012.

[53] Y.-J. Kim, H.-J. Park, G. Lee et al., "Neuroprotective effects of human mesenchymal stem cells on dopaminergic neurons through anti-inflammatory action," Glia, vol. 57, no. 1, pp. 13-23, 2009.

[54] N. K. Lee, J. Yang, E. H. Chang et al., "Intra-arterially delivered mesenchymal stem cells are not detected in the brain parenchyma in an Alzheimer's disease mouse model," PLoS One, vol. 11, no. 5, article e0155912, 2016.

[55] D. J. Selkoe, “Alzheimer's disease: genes, proteins, and therapy,” Physiological Reviews, vol. 81, no. 2, pp. 741-766, 2001.

[56] J. K. Lee, H. K. Jin, and J.-s. Bae, "Bone marrow-derived mesenchymal stem cells reduce brain amyloid- $\beta$ deposition and accelerate the activation of microglia in an acutely induced Alzheimer's disease mouse model," Neuroscience Letters, vol. 450, no. 2, pp. 136-141, 2009.

[57] J. Liu and L. Li, "Targeting autophagy for the treatment of Alzheimer's disease: challenges and opportunities," Frontiers in Molecular Neuroscience, vol. 12, p. 203, 2019.

[58] J. Y. Shin, H. J. Park, H. N. Kim et al., "Mesenchymal stem cells enhance autophagy and increase $\beta$-amyloid clearance in Alzheimer disease models," Autophagy, vol. 10, no. 1, pp. 32-44, 2014.

[59] H. J. Kim, S. W. Seo, J. W. Chang et al., "Stereotactic brain injection of human umbilical cord blood mesenchymal stem cells in patients with Alzheimer's disease dementia: a phase 1 clinical trial," Alzheimer's \& Dementia: Translational Research \& Clinical Interventions, vol. 1, no. 2, pp. 95-102, 2015.

[60] S. Forostyak, P. Jendelova, M. Kapcalova, D. Arboleda, and E. Sykova, "Mesenchymal stromal cells prolong the lifespan in a rat model of amyotrophic lateral sclerosis," Cytotherapy, vol. 13, no. 9, pp. 1036-1046, 2011.

[61] A. Uccelli, M. Milanese, M. C. Principato et al., "Intravenous mesenchymal stem cells improve survival and motor function in experimental amyotrophic lateral sclerosis," Molecular Medicine, vol. 18, no. 5, pp. 794-804, 2012.

[62] P. Petrou, Y. Gothelf, Z. Argov et al., "Safety and clinical effects of mesenchymal stem cells secreting neurotrophic factor transplantation in patients with amyotrophic lateral sclerosis," JAMA Neurology, vol. 73, no. 3, pp. 337-344, 2016.

[63] D. Krakora, P. Mulcrone, M. Meyer et al., "Synergistic effects of GDNF and VEGF on lifespan and disease progression in a familial ALS rat model," Molecular Therapy, vol. 21, no. 8, pp. 1602-1610, 2013. 
[64] D. Karussis, C. Karageorgiou, A. Vaknin-Dembinsky et al., "Safety and immunological effects of mesenchymal stem cell transplantation in patients with multiple sclerosis and amyotrophic lateral sclerosis," Archives of Neurology, vol. 67, no. 10 , pp. 1187-1194, 2010.

[65] C. Esopenko and B. Levine, "Aging, neurodegenerative disease, and traumatic brain injury: the role of neuroimaging," Journal of Neurotrauma, vol. 32, no. 4, pp. 209-220, 2015.

[66] F. Anbari, M. A. Khalili, A. R. Bahrami et al., "Intravenous transplantation of bone marrow mesenchymal stem cells promotes neural regeneration after traumatic brain injury," Neural Regeneration Research, vol. 9, no. 9, pp. 919-923, 2014.

[67] C. S. Cox Jr., J. E. Baumgartner, M. T. Harting et al., “Autologous bone marrow mononuclear cell therapy for severe traumatic brain injury in children," Neurosurgery, vol. 68, no. 3, pp. 588-600, 2011.

[68] J. Cox and S. Charles, Treatment of adult severe traumatic brain injury using autologous bone marrow mononuclear cells, Texas Univ Health Science Center at Houston, 2014.

[69] M. Matuskova, K. Hlubinova, A. Pastorakova et al., "HSV-tk expressing mesenchymal stem cells exert bystander effect on human glioblastoma cells," Cancer Letters, vol. 290, no. 1, pp. 58-67, 2010.

[70] F. S. Nouri, X. Wang, and A. Hatefi, "Genetically engineered theranostic mesenchymal stem cells for the evaluation of the anticancer efficacy of enzyme/prodrug systems," Journal of Controlled Release, vol. 200, pp. 179-187, 2015.

[71] C. Song, J. Xiang, J. Tang et al., "Thymidine kinase gene modified bone marrow mesenchymal stem cells as vehicles for antitumor therapy," Human Gene Therapy, vol. 22, no. 4, pp. 439449, 2011.

[72] B. C. Parker Kerrigan, T. Shahar, S. Yamashita, and F. F. Lang, "The role of mesenchymal stromal cells in human brain tumors," in The Biology and Therapeutic Application of Mesenchymal Cells, pp. 857-872, John Wiley \& Sons, Inc., 2016.

[73] N. G. Rainov, "A phase III clinical evaluation of herpes simplex virus type 1 thymidine kinase and ganciclovir gene therapy as an adjuvant to surgical resection and radiation in adults with previously untreated glioblastoma multiforme," Human Gene Therapy, vol. 11, no. 17, pp. 2389-2401, 2000.

[74] G. Almeida-Porada, A. J. Atala, and C. D. Porada, "Therapeutic mesenchymal stromal cells for immunotherapy and for gene and drug delivery," Molecular Therapy-Methods \& Clinical Development, vol. 16, pp. 204-224, 2020.

[75] K. Nakamura, Y. Ito, Y. Kawano et al., "Antitumor effect of genetically engineered mesenchymal stem cells in a rat glioma model," Gene Therapy, vol. 11, no. 14, pp. 1155-1164, 2004.

[76] A. Nakamizo, F. Marini, T. Amano et al., "Human bone marrow-derived mesenchymal stem cells in the treatment of gliomas," Cancer Research, vol. 65, no. 8, pp. 3307-3318, 2005.

[77] A. U. Ahmed, C. E. Rolle, M. A. Tyler et al., "Bone marrow mesenchymal stem cells loaded with an oncolytic adenovirus suppress the anti-adenoviral immune response in the cotton rat model," Molecular Therapy, vol. 18, no. 10, pp. 18461856, 2010.

[78] A. M. Sonabend, I. V. Ulasov, M. A. Tyler, A. A. Rivera, J. M. Mathis, and M. S. Lesniak, "Mesenchymal stem cells effectively deliver an oncolytic adenovirus to intracranial glioma," Stem Cells, vol. 26, no. 3, pp. 831-841, 2008.

[79] R. L. Yong, N. Shinojima, J. Fueyo et al., "Human bone marrow-derived mesenchymal stem cells for intravascular delivery of oncolytic adenovirus 24-RGD to human gliomas," Cancer Research, vol. 69, no. 23, pp. 8932-8940, 2009.

[80] J. Kiyokawa and H. Wakimoto, "Preclinical and clinical development of oncolytic adenovirus for the treatment of malignant glioma," Oncolytic Virotherapy, vol. 8, pp. 27-37, 2019.

[81] J.-C. Olivier, "Drug transport to brain with targeted nanoparticles," NeuroRX, vol. 2, no. 1, pp. 108-119, 2005.

[82] M. Roger, A. Clavreul, M.-C. Venier-Julienne et al., "Mesenchymal stem cells as cellular vehicles for delivery of nanoparticles to brain tumors," Biomaterials, vol. 31, no. 32, pp. 83938401,2010

[83] X. Wang, J.-Q. GAO, X. Ouyang, J. Wang, X. Sun, and Y. Lv, "Mesenchymal stem cells loaded with paclitaxel\&ndash;poly(lactic-co-glycolic acid) nanoparticles for glioma-targeting therapy," International Journal of Nanomedicine, vol. 13, pp. 5231-5248, 2018. 